\title{
Hybrid Multi-Pixel Array X-ray Detectors for Real-time Direct Detection of Hard X-rays
}

\author{
Hashini M. Thirimanne*, K. D. G. Imalka Jayawardena*, Andrew Nisbet, Yonglong Shen, R. M. \\ Indrachapa Bandara, Christopher A. Mills and S. Ravi P. Silva
}

\begin{abstract}
X-ray detectors currently employed in dosimetry suffer from a number of drawbacks including the inability to conform to curved surfaces and being limited to smaller dimensions due to available crystal sizes. In this study, a hybrid X-ray detector (HXD) has been developed which offers real-time response with added advantages of being highly sensitive over a broad energy range, mechanically flexible, relatively inexpensive and able to be fabricated over large areas on the desired surface. The detector comprises of an organic matrix embedded with high atomic number inorganic nanoparticles which increase the radiation attenuation and within the device allows for simultaneous transfer of electrons and holes. The HXD delivers a peak response of 14 $\mathrm{nA} \mathrm{cm}{ }^{-2}$, which corresponds to a sensitivity of $58 \mu \mathrm{C} \mathrm{mGy}^{-1} \mathrm{~cm}^{-3}$, under the exposure of $15 \mathrm{MV}$ hard X-rays generated by a medical linear accelerator. The angular dependence of the HXD has been studied, which offers a maximum variation of $26 \%$ in the posterior versus lateral beam directions. The flexible HXD can be conformed to the human body shape and is expected to eliminate variations due to source-to-skin distance with reduced physical evaluation complexities.
\end{abstract}

Index Terms - Hard X-rays, Hybrid X-ray detector, LINAC, Optoelectronic devices.

\section{INTRODUCTION}

$\mathrm{D}^{2}$ ETECTION of ionizing radiation is a priority in radiation protection, radiotherapy quality assurance (QA) and in medical applications involving imaging and determination of dose distributions for radiotherapy. The latter case is widely used in radiation oncology, which mainly uses linear particle accelerators (LINAC) to provide precision targeting of a tumour from a beam of X-rays. During the past 60 years, medical LINACs have evolved through many generations, where today, high precision radiotherapy technologies have increased with the emergence of volumetric-modulated radiation therapy (VMAT) and stereotactic body radiation therapy (SBRT).[1] Individual treatment verification is gaining attention in such high-precision radiotherapy, where accurate dose delivery to a patient is essential.

At present the detection of ionizing radiation is achieved by high-resolution flat panel arrays, radiographic films, diode arrays and ionization chambers, where each have their own advantages and disadvantages for specific applications.[2] Radiographic films or self-developing films, such as GafChromic films, can serve as a radiation detector, a relative dosimeter, a display device and an archival medium. They are thin, flexible and can be cut to fit various

The paper was submitted for review on XX/XX/2019. This work was supported by the Leverhulme Trust through research project grant No. RPG2014-312. *These authors contributed equally to this work.

H. M. Thirimanne, K. D. G. I. Jayawardena, R. M. I. Bandara, C. A. Mills, S. R. P. Silva are with the Advanced Technology Institute, University of Surrey, Guildford, Surrey, GU2 7XH UK (e-mail: h.m.thirimanne@surrey.ac.uk,i.jayawardena@surrey.ac.uk,i.rajapakshe@s urrey.ac.uk, c.mills@ surrey.ac.uk, s.silva@surrey.ac.uk ). geometries and are relatively inexpensive. However, they do not offer real-time dosimetry, and require careful calibration against ionization chamber measurements.[3],[4] On the other hand, flat panel or diode arrays offer high sensitivity with an instant read-out, but are less useful for beam calibration due to their possible tendency to damage from accumulated dose. Further, they are rigid, heavy and bulky. However, compared to gas filled detectors, the sensitivity of solid state detectors is about $10^{4}$ times higher, due to the lower average energy required to produce an ion pair in solid detector materials compared with air, hence allowing for miniaturization of solid state radiation monitoring instruments.[2] Hence, a combination between the radiographic film and a diode array would be an ideal candidate, offering a range of qualities such as the detector conformability to different geometries while offering a realtime response. This can be achieved by organic-inorganic hybrid optoelectronic devices.

The past three decades have shown an immense interest in the use of organic semiconductor materials for optoelectronic devices. In addition, many groups have explored the use of organic semiconductors as detectors for soft X-rays, which could potentially lead to applications such as, imaging, non-destructive testing and security. The most

A. Nisbet is with the Department of Physics, University of Surrey, Guildford, Surrey, GU2 7XH UK and Department of Medical Physics, Royal Surrey County Hospital NHS Foundation Trust, Egerton Road, Guildford, GU2 7XX, UK (e-mail: a.nisbet@surrey.ac.uk). Y. Shen is with the School of Materials Science and Engineering, Zhengzhou University, No. 100 Science Avenue, Zhengzhou City, Henan Province P.R.China. 450001. (e-mail: shenyonglong@zzu.edu.cn) 
widely explored devices rely on indirect detection of X-rays which comprises of a photodiode coupled to a scintillator [5],[6],[7], or the insertion of scintillators in to an organic matrix itself [8]. Alternatively, direct detection of X-rays has been explored using thick films of conjugated polymers [9], organic semiconductor single crystals [10] and perovskite thin films [11], perovskite single crystals [12],[13] or by sintered perovskite wafers [14].

However, limited work has been reported on the performance of the organic semiconductors under X-ray energies of 1-15 MV range; which will be termed as 'hard Xrays' in this study.[5],[15] Here we demonstrate the use of an organic-inorganic hybrid material as a hybrid X-ray detector (HXD) for the direct detection of hard X-rays produced from a medical LINAC. The performance of this system under soft X-rays $(\sim 50 \mathrm{kV})$ has been previously reported.[16],[17] The inclusion of high atomic number (Z) nanoparticles (NPs) enables the attenuation of incoming Xrays while directly generating electron-hole charge carriers via the Compton effect. The polymer-based detectors with the inclusion of high $\mathrm{Z}$ NPs have been reported previously,[18] which mostly act as photoconductors as they transport only a single charge carrier with the aid of the single polymer material. A balanced electron-hole mobility is essential for an ideal detector in order to extract all the generated charge carriers, without trapping one of them within the detector and creating space charge effects. Hence, the developed detector in this work is based on an organic bulk heterojunction (BHJ) which allows both generated electron and holes to be collected. A similar 'triple junction' concept with hybrid carbon nanotubes embedded with an organic photovoltaic structure also showed enhanced efficiency in its operation.[19] The detector developed herein, shows a high sensitivity of $58 \mu \mathrm{C} \mathrm{mGy}^{-1} \mathrm{~cm}^{-3}$ at 6 $\mathrm{MV}$, and a linear response over a wide range of $\mathrm{X}$-ray doses.

Concerns could arise, on whether organic semiconductors can withstand a significant dose of MV X-rays without undergoing degradation or suffering performance degradation by the generation of charge traps. However, a number of studies have now demonstrated the stability of organics under $\mathrm{X}$-rays, demonstrating the potential applicability of this technology for radiation oncology. [20], [21]

\section{METHODS AND PROCEDURES}

\section{A. Material Preparation}

The X-ray sensitive active material was prepared by dissolving regioregular poly(3-hexylthiophene-2,5-diyl) (P3HT, $40 \mathrm{mg}$, Rieke $4002 \mathrm{EE}$ ) and [6,6]-Phenyl $\mathrm{C}_{71}$ butyric acid methyl ester ( $\mathrm{PC}_{70} \mathrm{BM}, 40 \mathrm{mg}, 99 \%$ pure; Solenne) in dichlorobenzene $(1 \mathrm{ml})$ to produce a $\mathrm{P} 3 \mathrm{HT}: \mathrm{PC}_{70} \mathrm{BM}$ solution. Bismuth oxide $\left(\mathrm{Bi}_{2} \mathrm{O}_{3}\right)$ NPs were dispersed in the $\mathrm{P} 3 \mathrm{HT}: \mathrm{PC}_{70} \mathrm{BM}$ solution to give a $\mathrm{Bi}_{2} \mathrm{O}_{3}$ concentration of 40 $\mathrm{mg} \mathrm{ml}^{-1}$.

\section{B. Device Fabrication}

Devices were fabricated on ITO $\left(\operatorname{In}_{2} \mathrm{O}_{3}: \mathrm{Sn}\right)$ glass substrate $\left(15 \mathrm{~mm} \times 15 \mathrm{~mm}, 10 \mathrm{ohms} \square^{-1}\right.$, Luminescence Technology Corp.). A hole transporting and electron blocking Poly(3,4ethylenedioxythiophene)-poly(styrenesulfonate)

(PEDOT:PSS; P VP Al 4083; Heraeus) layer was spin coated in air $(5000 \mathrm{rpm}$ for $40 \mathrm{~s})$ and annealed at $150{ }^{\circ} \mathrm{C}$ for 10 minutes to give a thickness of $40 \mathrm{~nm}$. The prepared X-ray sensitive active material was then cast $(90 \mu \mathrm{l})$ giving an active layer thickness of $20 \mu \mathrm{m}$. Two stage annealing was followed to obtain a crystalline film. Firstly, a low temperature annealing process was followed at $60{ }^{\circ} \mathrm{C}$ for 40 minutes inside an oven and secondly the device was annealed at $140{ }^{\circ} \mathrm{C}$ for 10 minutes in a $\mathrm{N}_{2}$ glove box (MBraun MB20G). Devices were kept under vacuum at a pressure less than $3 \times 10^{-6}$ mbar for 24 hours to remove any residual solvent. This was followed by deposition of the 1-2,9dimethyl-4,7-diphenyl-1,10-phenanthroline (BCP; sublimed grade, Sigma Aldrich, 99.99\% purity; thickness of $5 \mathrm{~nm}$ ) which is a hole blocking, electron transporting layer, followed by deposition of an $\mathrm{Al}$ cathode $(150 \mathrm{~nm})$ by thermal evaporation. Device encapsulation was carried out with the deposition of UV light cure adhesive glue $(20 \mu$ l of encapsulation epoxy; Ossila Ltd.) pressed with an encapsulation glass slide (Ossila Ltd.) and the epoxy UV cured for 5 minutes.

The flexible pixelated array was fabricated on Kapton substrates $(50 \mu \mathrm{m})$, with patterned chromium $(10 \mathrm{~nm}) /$ gold $(\mathrm{Au} ; 100 \mathrm{~nm})$ bilayer contact deposited by evaporation as the anode. X-ray sensitive material $(1.44 \mathrm{ml})$ was casted and the rest of the procedure mentioned above followed. The flexible device was not encapsulated. Pixel size is $1.1 \times 1.1 \mathrm{~cm}^{2}$ with a pixel pitch of $1 \mathrm{~mm}$. X-ray measurements were carried out on 6 pixels of the flexible array.

\section{Structural Characterisation}

TEM was performed using a FEI G2 F20 transmission electron microscope (FEI, Thermal-Fisher) operated at $200 \mathrm{kV}$. TEM samples were prepared by using Ion Milling (Gatan, Model 691).

\section{Thermogravimetric analysis}

To investigate the weight $\%$ of $\mathrm{Bi}_{2} \mathrm{O}_{3}$ present in the device, thermogravimetric analysis (TGA) was carried out under air using Q500, TA Instruments. The sample was decomposed from room temperature to $700^{\circ}$ with a heating rate of $10^{\circ} \mathrm{C}$ $\min ^{-1}$.

\section{X-RAY MEASUREMENTS}

A multi-mode linear accelerator (Clinac iX Varian, USA) located at the Royal Surrey County Hospital NHS Foundation Trust was used as a hard X-ray irradiation source. For the dose rate dependency experiment, 6 and 15 MV X-rays were used with dose rates ranging from 100 to $400 \mathrm{cGy} \mathrm{min}^{-1}$. In all experiments, the HXD was irradiated at the beam central axis with source-surface distance (SSD) of $100 \mathrm{~cm}$ and with $2 \times 2 \mathrm{~cm}^{2}$ field size. For the dose dependency experiment, a field size of $10 \times 10 \mathrm{~cm}^{2}$ was used. $\mathrm{X}$-rays were irradiated to the device through the ITO or Au 
(for the pixelated array) contact. A Keithley 2400 source measurement unit (SMU) was used for recording the electrical characteristics of the devices and three measurements were obtained for each experiment.

The dependence of the diode sensitivity upon the angle of beam incidence, with respect to the longitudinal axis of the detector head, was measured using a $6 \mathrm{MV}$ photon beam with a dose rate of $400 \mathrm{cGy} \mathrm{min}{ }^{-1}$. The SSD was $100 \mathrm{~cm}$ and the linear accelerator gantry was moved from the vertical down direction $\left(0^{\circ}\right)$ to $30^{\circ}, 60^{\circ}$ and $90^{\circ}$ in both clockwise and counter clockwise directions. The angular dependency was measured using the same HXD, and three measurements were obtained for each investigated angle.

Flexible pixelated array was irradiated with $6 \mathrm{MV}$ X-rays where the response of the X-ray irradiation was monitored while having the pixel array both under flat and flexed conditions.

\section{RESUlTS AND DISCUSSION}

The HXD has a sandwich-type architecture consisting of

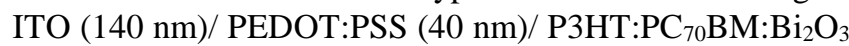
$(20 \mu \mathrm{m}) / \mathrm{BCP}(5 \mathrm{~nm}) / \mathrm{Al}(150 \mathrm{~nm})$ (Fig. 1a, b).

$\mathrm{P} 3 \mathrm{HT}: \mathrm{PC}_{70} \mathrm{BM}$ is an interpenetrating $\mathrm{BHJ}$ network, consisting of nanoscale diodes throughout the active layer, allowing the transport of both holes and electrons separately via $\mathrm{P} 3 \mathrm{HT}$ and $\mathrm{PC}_{70} \mathrm{BM}$ respectively. In this study, we have utilized $\mathrm{Bi}_{2} \mathrm{O}_{3} \mathrm{NPs}$, due to the high atomic mass of $\mathrm{Bi}(\mathrm{Z}=83)$ in order to increase the $\mathrm{X}$-ray attenuation. Under hard $\mathrm{X}$ rays, direct $\mathrm{X}$-ray photocurrent generation from the bulk high $\mathrm{Z}$ materials occurs primarily through Compton scattering.[22] Fig. 1c shows a TEM image of the presence of nanoparticles in the active layer exhibiting a good distribution of NPs. We have previously reported that such BHJ and NP combination has the potential to lead to NP aggregation on the active layer surfaces [16], but such metal electrode structuring leads to efficient charge extraction through enhancements in the electric field [23].

The dark current density, an important detector metric, was observed to be $\sim 10^{-6} \mu \mathrm{A} \mathrm{cm}^{-2}$ at $-20 \mathrm{~V}$, and the dark current density of the HXD is comparable with a device without any NPs (Fig. 1d). The rectification of the HXD is greater than the NP free reference, concluding that the addition of NPs has made the device more conducting despite the lower dark current measured in Fig. 1 (d) under reverse bias conditions. For industrial relevance the range of the dark current should be sub $\mathrm{nA} \mathrm{cm}^{-2}$, while the HXD offers dark currents of 6 and $41 \mathrm{nA} \mathrm{cm}^{-2}$ at -0.1 and $-1 \mathrm{~V}$ respectively. The resulting inbuilt depletion region, with local electric fields as high as $\sim 200 \mathrm{~V} \mu \mathrm{m}^{-1}$, is further enhanced by dielectric inhomogeneities [24],[25] in the material enabling efficient electron and hole extraction from the entirety of the depleted active layer. This allows one to obtain a high X-ray response even under low applied voltages.

TGA was performed to confirm the weight percentage of $\mathrm{Bi}_{2} \mathrm{O}_{3}$ NPs in the active layer of the HXD (Fig. 1e). A device with only P3HT: $\mathrm{PC}_{70} \mathrm{BM}$ has been analysed as a reference, which delivers a 0 weight $\%$ confirming the full combustion of the organic material beyond $600{ }^{\circ} \mathrm{C}$. The $\mathrm{Bi}_{2} \mathrm{O}_{3}$ weight $\%$ in the HXD was found to be $45 \%$. Further, the significant double knee type behaviour seen in the reference curve is known to be due to the addition of fullerene $\left(\mathrm{C}_{70}\right)$.
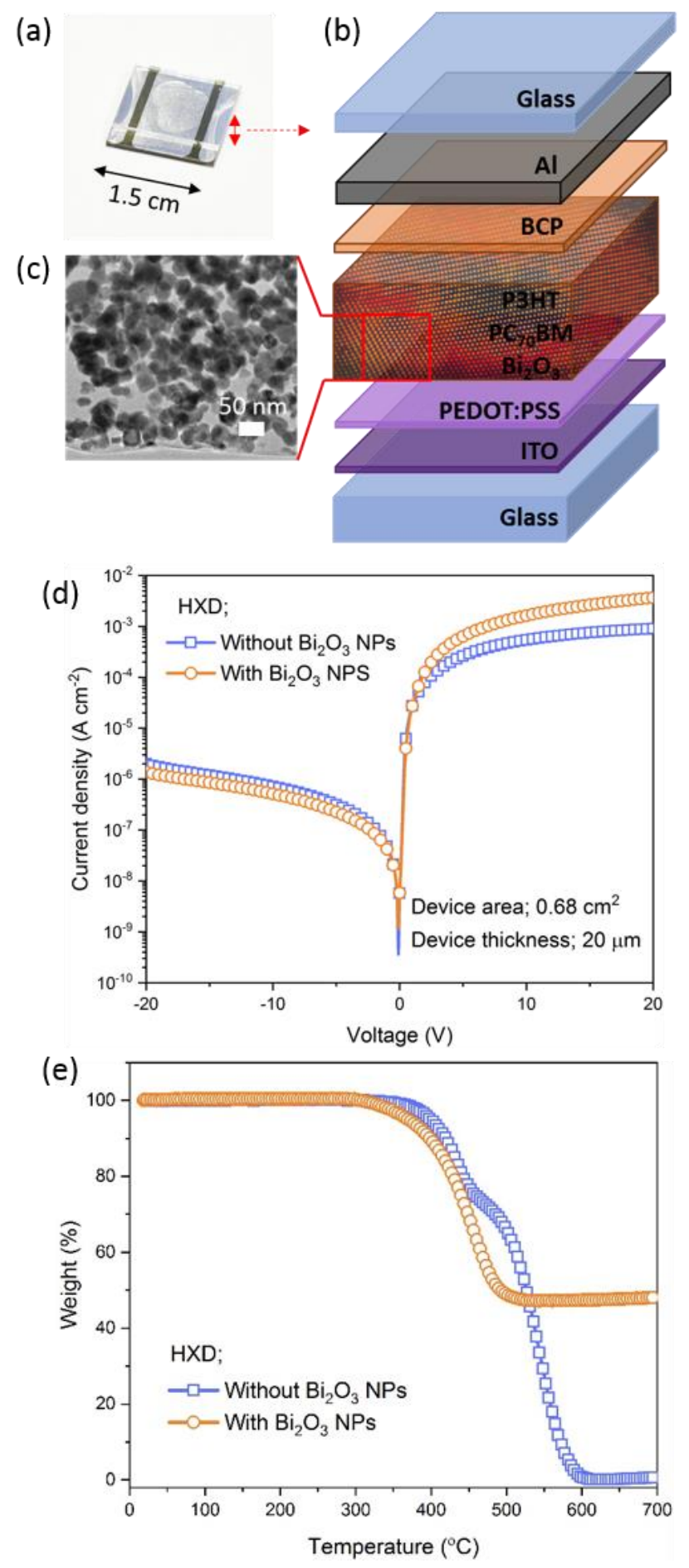

Fig. 1. (a) The top view of the device. (b) Device schematic structure. (c) A cross sectional TEM image showing the distribution of NPs. (d) Dark current density-voltage response of devices with and without $\mathrm{Bi}_{2} \mathrm{O}_{3} \mathrm{NPs}$. (e) TGA analysis of the active material film with and without $\mathrm{Bi}_{2} \mathrm{O}_{3} \mathrm{NPs}$.

\section{A. Dose Rate Dependency}

The raw data of the HXD when exposed to 6 MV X-rays 
under a dose rate of $454 \mathrm{mGy} \mathrm{s}^{-1}$, recorded by the source measure unit (SMU) is presented in Figure 2(a). Here, the HXD was kept under dark conditions for two minutes and the X-ray irradiation was initiated at $t=120 \mathrm{~s}$ and exposed to $\mathrm{X}$-rays for 30 seconds. Such 30 second exposures were given at one-minute intervals (LINAC ON/OFF 3 cycles) to investigate the repeatability of the performance of the detector. It is noted that the dark current is very high and a heavy exponential decay in the drift of the dark current was observed. In order to obtain a high signal-to-noise ratio, the dark current should be minimised to detect signals under low radiation doses. Thermally generated charge carriers, charge injection from the contact, charge recombination and defects in the bulk and interfaces of the organic semiconductor should be very well controlled in order to reduce the dark current. Further, traps can also act as an origin for high dark current, where the presence of oxygen and moisture lead to shallow trap levels and morphological ordering and air exposure strongly influence deep trap concentration.[26]

The response under $\mathrm{X}$-rays has been extracted by subtracting the dark current and is presented in Fig. 2b. A peak response current density of $14 \mathrm{nA} \mathrm{cm}^{-2}$ was achieved after the baseline correction (dotted red line in Fig. 1a). The device was also exposed to 6 and 15 MV LINAC X-ray beam energies for different dose rates (Fig. 2c) and offers good dose rate linearity, demonstrating that the detector developed is a suitable dosimeter for accurate dose measurement.

Under a $114 \mu \mathrm{Gy} \mathrm{s}^{-1}$ dose rate and $-10 \mathrm{~V}$ reverse bias, the detector delivered a sensitivity of 30 and $58 \mu \mathrm{C} \mathrm{mGy}^{-1} \mathrm{~cm}^{-3}$ for 6 and $15 \mathrm{MV} \mathrm{X}$-rays respectively, which can be calculated using,

$$
S=\frac{\int\left[I_{X-r a y}(t)-I_{d a r k}\right] d t}{D \times V}
$$

Here, $I_{X-\text { ray }}$ and $I_{\text {dark }}$ are the currents under X-ray irradiation and in the dark respectively, $D$ is the dose and $V$ is the detector volume. We note that these sensitivities are nearly $\times 100$ higher than those reported for organic photodetectors tested under 6 and $15 \mathrm{MV}$ LINAC using $\mathrm{Gd}_{2} \mathrm{O}_{2} \mathrm{~S}$ :Tb as scintillators.[5],[27]

\section{A. Angular Dependency}

The form of the human body (three dimensional in nature) results in a requirement in radiotherapy to vary the angle of incidence of the X-ray beam depending on the topography and morphology of the region under irradiation. Commercially available diode detectors are found to have as much as a $\pm 21 \%$ change in sensitivity with an increased radiation angle of incidence up to $90^{\circ}$ [28],[29], which creates additional complexity during the dose verification stage of patient treatment. Similarly, significant scattered dose from surfaces adjacent to the irradiation area can also reduce the accuracy of the dose delivered, which limits the use of this type of diode in any type of arc therapy. Therefore, minimisation of angular dependency is critical in semiconductor detector technologies.
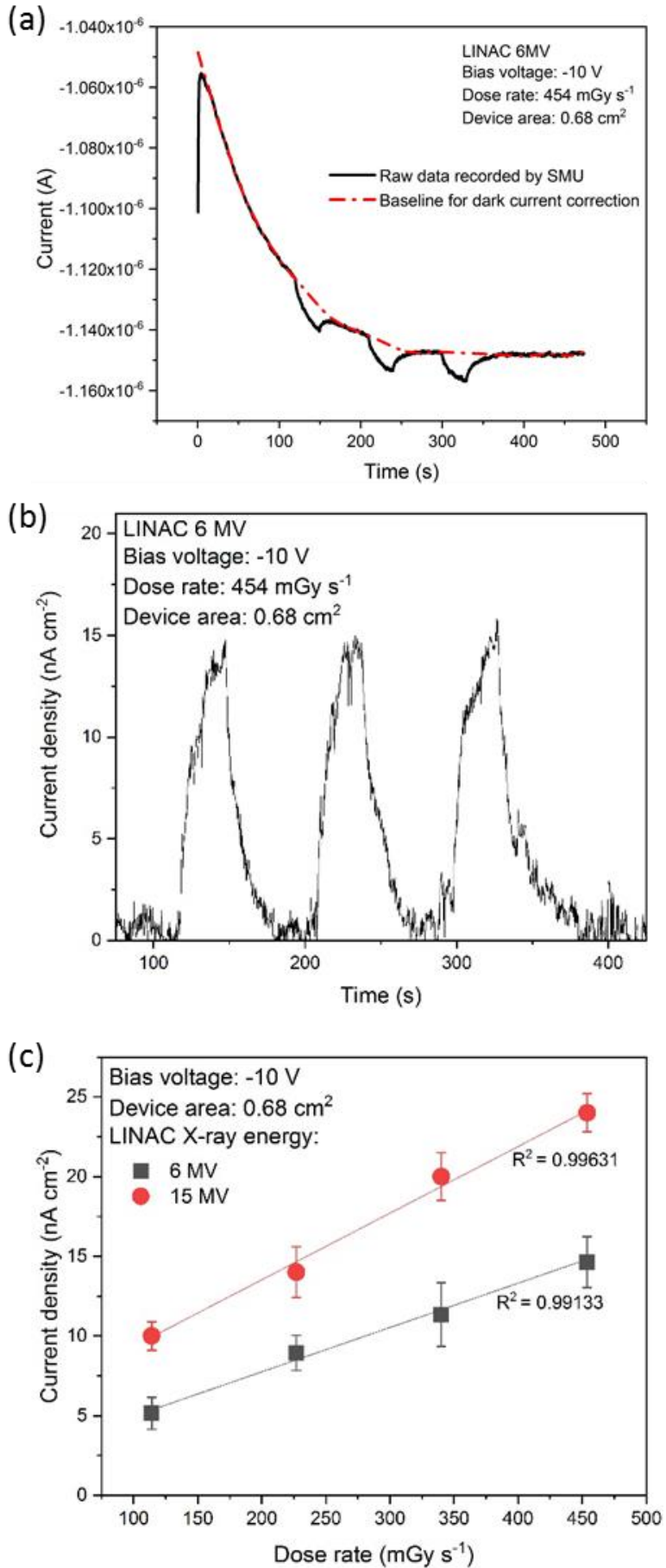

Fig. 2. (a) The raw data of the HXD irradiated under 6 MV LINAC hard Xrays and (b) the dark current subtracted repetitive performance of the HXD. (c) The dose dependency of the HXD under X-ray irradiation of 6 and 15 MV LINAC.

Fig. 3 shows the angular dependence of the HXD irradiated with $6 \mathrm{MV}$ photons. The response was normalised to the reading at $0^{\circ}$. In Fig. $3 \mathrm{~b}$, each data point represents an average value and one standard deviation of 3 measurements. The difference in the HXD response is within 19\% and 26\% for the angle ranging from $0^{\circ}$ to $+90^{\circ}$ and $-90^{\circ}$ respectively. Consequently, for a planar detector, the $\mathrm{Bi}_{2} \mathrm{O}_{3}$ loaded polymers show an equivalent angular dependency to that produced by a commercial silicon reference diode. 

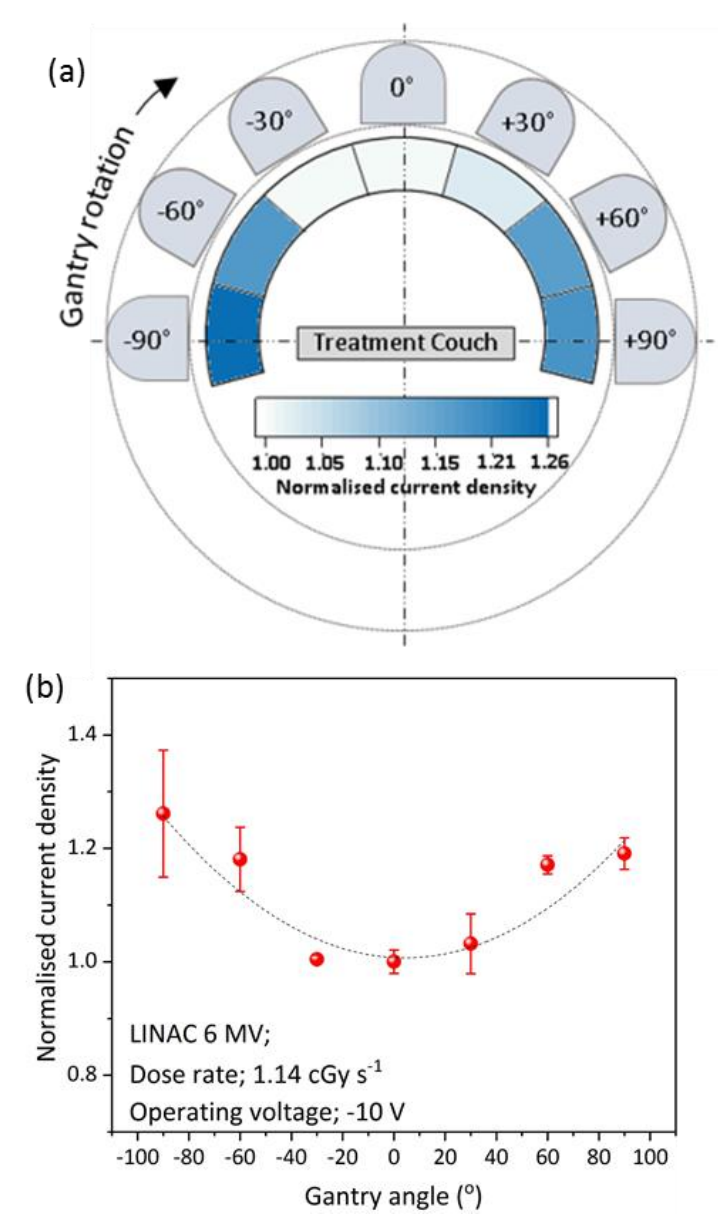

Fig. 3. (a) Schematic diagram of the angular variation of the LINAC and the performance of the HXD. The gantry was rotated for $30^{\circ}, 60^{\circ}, 90^{\circ},-30^{\circ}$, $60^{\circ}$ and $-90^{\circ}$. (b) The normalized current densities of the HXD under different gantry positions. Three measurements were taken per condition and the dotted line fit is the parabolic fit to the represented data.

The geometry of the detector is considered to be a critical factor in its angular dependant behaviour. Studies of similar angular dependence observed for a variety of detectors such as Matrixx (Iba Dosimtery America, Inc., Bartlett, TN)[30], glass beads[31], MOSkin, Si diode PTW 60008[32], nanoDot optically stimulated luminescent detector (OSLD)[33], radioluminescent glass rod dosimeters [34] etc. where the detectors comprise of different geometries such as cubic[30], rods[34] and spheres[31] have indicated a variation in the angular dependence.[33] The detectors also have different read-out systems, where some are stand-alone detectors such as thermo luminescent detectors (TLD), OSLD and others employ electronics (surface diodes). Jursinic reports [35] comparisons of the angular dependency between surface diode, TLD and OSLD, where the TLD and OSLD show almost no angular dependence, with uncertainty around $1 \%$, while the surface diode has a complicated dependency on the incident angle of the radiation with a $21 \%$ drop in response with angle. This could possibly be due to the TLD and OSLD technologies being based on 3D crystal systems, while the diode and polymer systems are quasi-2D.

The HXD presented in this work is a planar X-ray sensitive detector which, on a macroscopic scale, creates an irregular response at various angles as viewed by the incoming photon beam. The design of the detector is also asymmetric, with differences between the front and back electrodes. Further, the detector is connected to the Keithley via a $3 \mathrm{M}$ SOIC test clip consisting of gold pins connected to one side of the diode. It could be hypothesised that the variation of the angular dependence seen in Fig. 3 is a result of asymmetry in the mounting of the diode, where the low energy electrons can be scattered due to the Au metal test clip positioned on the side of the detector. This could possibly explain the high sensitivity when the beam angle is at $\pm 90^{\circ}$.

The observed angular dependence may be important for a wide range of applications which include skin dose measurements on patients and patient treatment field dosimetry QA tests, specifically for multifield treatments where some beams could be at an angle at or near perpendicular to the dosimeter. The findings of this study do not exclude the detector from being used for these applications, especially considering the advantages of the detector, but there is a need for a correction factor when accounting for the treatments at different angles.

There is precedence for the use of edge-on detectors for X-ray detection incident to the beam.[36],[37],[38] Typically, a small number of crystal-based (e.g. silicon, CZT) detectors will be arranged with an edge-on architecture to maximise X-ray capture sensitivity. The possibility of producing the hybrid detectors described here on thin, flexible plastic substrates which can be folded or arranged in a multi-leaf configuration allows for detection in a variety of edge-on configurations. Alternatively, angular dependence can be mitigated for when using a flexible pixelated detector by conformal arrangement into a tubular or spherical structure, reducing the angular dependence and simplifying medical dosimetry planning, but potentially sacrificing detector sensitivity.

\section{B. Pixel Array}

The major advantage of the organic-inorganic HXD is the ability to wrap the detector around a body part to measure the surface dose accurately. As the HXD is thin, conformable and can be fabricated in large area as needed, it is ideal for applications in which the detector needs to be conformally arranged on a patient or anthropomorphic phantom. Here, as a preliminary study, we show a flexible array (FA) mounted on an anthropomorphic Rando phantom to primarily investigate the difference of dose distribution using a flat and a flexed detector (Fig. 4a).

As shown in Fig. $4 \mathrm{~b}$, the schematic FA comprises of 6 pixels, where the region of interest (ROI) is an area of $4 \mathrm{~cm}^{2}$ which has been exposed to $6 \mathrm{MV}$ X-rays. The ROI interacts with the two central pixels in the FA. The array of sensors is not used here to determine the pixel-pixel variation, but rather to characterise the spatial dose distribution. The pixel size and the pixel pitch can be easily adjusted according to the required area size or application. 
(a)
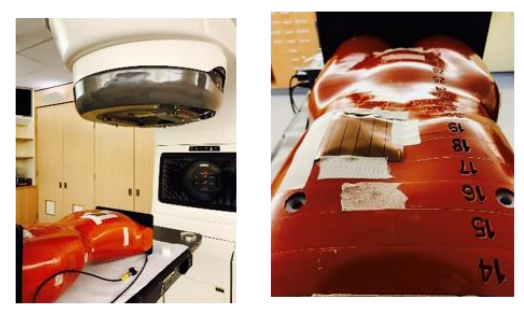

(b)

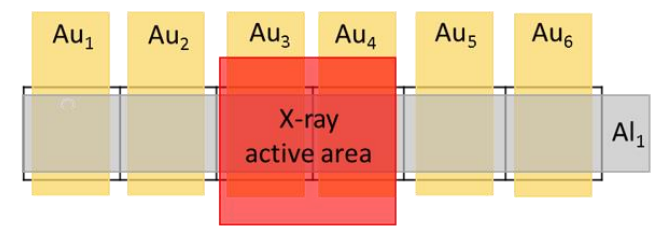

(c)

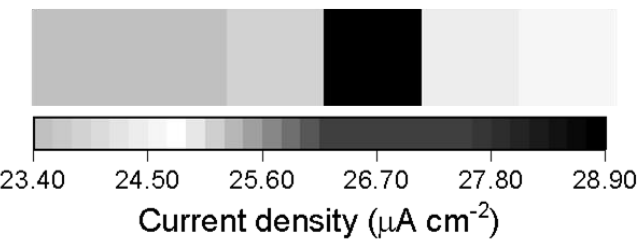

(d)



(e)

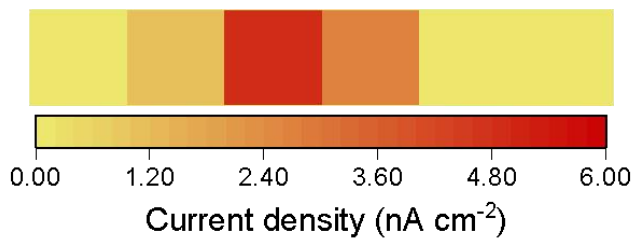

Fig. 4. (a) HXD mounted on an anthropomorphic phantom to exhibit its conformability according to the curved body parts. (b) The schematic of the pixel array, containing 6 pixels where the region of interest is indicated by the red square. (c) The variation of the dark current densities of the pixel array. The response of each pixel under $6 \mathrm{MV}$ X-rays when the detector is (d) flat and (e) flexed (as demonstrated in a).

Initially, the dark current density of each pixel in the strip array was measured, to evaluate the variation of the response which might arise due to the leakage of the diodes (Fig. 4c). The dark current densities of the pixels are $\sim 25 \mu \mathrm{A} \mathrm{cm}^{-2}$, and the leakage current variation can be considered as negligible among the pixels. Secondly, the dose of the central and peripheral pixels was measured individually while the FA is positioned on a flat surface (Fig. 5d). The response of the FA has been corrected for the dark current produced by the respective pixel. In this case, only the irradiated pixels at the ROI have responded significantly to the incoming $\mathrm{X}$-ray photons, while the output current density of $\mathrm{Au}_{3}-\mathrm{Al}_{1}$ pixel is slightly higher than the $\mathrm{Au}_{4}-\mathrm{Al}_{1}$ pixel. The variation between the two pixels could possibly be due to the variation of the quantity of NPs present in the active volume. This could be easily addressed by optimising the coating condition to have an approximately even quantity of NPs throughout the entire matrix.
Fig. $4 \mathrm{e}$ is the response of the FA mounted on the chest of the anthropomorphic phantom, where the FA and ROI is distorted compared to the flat surface. Interestingly the response of the $\mathrm{Au}_{3}-\mathrm{Al}_{1}$ has increased by $25 \%$ compared to the response of the detector in the flat position, and an additional response is detected from the $\mathrm{Au}_{2}-\mathrm{Al}_{1}$ pixel. A higher dose has been received at the uppermost point of the flexed detector. Further investigation is certainly needed to study the effect of the detector under flat and flexed configurations to monitor the effect of radiation dose distribution. In this study, this is a preliminary test performed to discuss the benefit of a flexible detector over a flat surface detector on curved surfaces to measure the spatial dose distribution around the ROI.

\section{CONCLUSION}

The HXD developed here offers a sensitivity response of $58 \mu \mathrm{C} \mathrm{mGy}{ }^{-1} \mathrm{~cm}^{-3}$ under $6 \mathrm{MV}$ hard X-rays generated by a medical linear accelerator. The detector is capable of directly detecting the incoming radiation offering a real-time response. The angular dependence of the HXD results in a maximum variation of $26 \%$, comparing the posterior versus lateral incoming beams, a value similar to that found for planar Silicon detectors. Production of a flexible, large area pixel detector allows the spatial dose distribution to be measured conformally on an anthropomorphic phantom, making the detector suitable for determining the radiation dose on the surface of a patient for in vivo dosimetry. The HXD also could be used by mounting on the patient for real time dosimetry during treatment.

\section{ACKNOWLEDGMENT}

The authors gratefully acknowledge support for this work from the Leverhulme Trust through a research project grant (No. RPG-2014-312), the Alan Roger's Innovation Award, University of Surrey and partial support obtained via NIHR. The authors thank V. Doukova (University of Surrey) for obtaining TGA results.

\section{REFERENCES}

[1] M. Teoh, C. H. Clark, K. Wood, S. Whitaker, and A. Nisbet, "Volumetric modulated arc therapy: a review of current literature and clinical use in practice," Br. J. Radiol., vol. 84, no. 1007, pp. 967-996, Nov. 2011.

[2] E. B. Podgorsak, "Radiation Oncology Physics: A Handbook for Teachers and Students."

[3] A. L. Palmer, A. Dimitriadis, A. Nisbet, and C. H. Clark, "Evaluation of Gafchromic EBT-XD film, with comparison to EBT3 film, and application in high dose radiotherapy verification," Phys. Med. Biol., vol. 60, no. 22, pp. 8741-8752, Nov. 2015.

[4] A. L. Palmer, D. A. Bradley, and A. Nisbet, "Evaluation and mitigation of potential errors in radiochromic film dosimetry due to film curvature at scanning," J. Appl. Clin. Med. Phys., vol. 16, no. 2, pp. 425-431, Mar. 2015.

[5] J. W. Kingsley, A. J. Pearson, L. Harris, S. J. Weston, and D. G. Lidzey, "Detecting 6MV X-rays using an organic photovoltaic device," Org. Electron., vol. 10, no. 6, pp. 1170-1173, Sep. 2009. 
[6] T. Agostinelli, M. Campoy-Quiles, J. C. Blakesley, R. Speller, D. D. C. Bradley, and J. Nelson, "A polymer/fullerene based photodetector with extremely low dark current for x-ray medical imaging applications," Appl. Phys. Lett., vol. 93, no. 20, p. 203305, Nov. 2008.

[7] P. E. Keivanidis et al., "X-ray stability and response of polymeric photodiodes for imaging applications," Cit. Appl. Phys. Lett, vol. 92, 2008.

[8] P. Büchele et al., "X-ray imaging with scintillator-sensitized hybrid organic photodetectors," Nat. Photonics, vol. 9, no. 12, pp. 843-848, Nov. 2015.

[9] A. Intaniwet, C. A. Mills, M. Shkunov, H. Thiem, J. L. Keddie, and P. J. Sellin, "Characterization of thick film poly(triarylamine) semiconductor diodes for direct x-ray detection," J. Appl. Phys., vol. 106, no. 6, p. 064513, Oct. 2009

[10] B. Fraboni et al., "Organic semiconducting single crystals as next generation of low-cost, room-temperature electrical X-ray detectors.," Adv. Mater., vol. 24, no. 17, pp. 2289-93, May 2012.

[11] S. Yakunin et al., "Detection of X-ray photons by solutionprocessed lead halide perovskites," Nat. Photonics, vol. 9, no. 7, pp. 444-449, May 2015.

[12] S. Yakunin et al., "Detection of gamma photons using solutiongrown single crystals of hybrid lead halide perovskites," Nat. Photonics, vol. 10, no. 9, pp. 585-589, Jul. 2016.

[13] W. Wei et al., "Monolithic integration of hybrid perovskite single crystals with heterogenous substrate for highly sensitive X-ray imaging," Nat. Photonics, vol. 11, no. 5, pp. 315-321, Apr. 2017.

[14] S. Shrestha et al., "High-performance direct conversion X-ray detectors based on sintered hybrid lead triiodide perovskite wafers," Nat. Photonics, vol. 11, no. 7, pp. 436-440, Jun. 2017.

[15] C. A. Mills et al., "Direct detection of 6 MV x-rays from a medical linear accelerator using a semiconducting polymer diode.," Phys. Med. Biol., vol. 58, no. 13, pp. 4471-82, Jul. 2013.

[16] H. M. Thirimanne et al., "High sensitivity organic inorganic hybrid X-ray detectors with direct transduction and broadband response," Nat. Commun., vol. 9, no. 1, p. 2926, Dec. 2018.

[17] K. D. G. I. Jayawardena et al., "Millimeter-Scale Unipolar Transport in High Sensitivity Organic-Inorganic Semiconductor X-ray Detectors," ACS Nano, vol. 13, no. 6, pp. 6973-6981, Jun. 2019.

[18] A. Intaniwet, C. A. Mills, M. Shkunov, P. J. Sellin, and J. L. Keddie, "Heavy metallic oxide nanoparticles for enhanced sensitivity in semiconducting polymer x-ray detectors.," Nanotechnology, vol. 23, no. 23, p. 235502, Jun. 2012.

[19] N. A. Nismy, K. D. G. I. Jayawardena, A. A. D. T. Adikaari, and S. R. P. Silva, "Photoluminescence quenching in carbon nanotube-polymer/fullerene films: carbon nanotubes as exciton dissociation centres in organic photovoltaics.," Adv. Mater., vol. 23, no. 33, pp. 3796-800, Sep. 2011.

[20] P. E. Keivanidis et al., "X-ray stability and response of polymeric photodiodes for imaging applications," Cit. Appl. Phys. Lett, vol. 92, 2008.

[21] J. W. Kingsley, S. J. Weston, and D. G. Lidzey, "Stability of XRay Detectors Based on Organic Photovoltaic Devices," IEEE J. Sel. Top. Quantum Electron., vol. 16, no. 6, pp. 1770-1775, Nov. 2010.

[22] G. F. Knoll, Radiation Detection and Measurement. John Wiley \& Sons, 2010.

[23] H. M. Thirimanne, K. D. G. I. Jayawardena, J. V. Anguita, V Stolojan, C. A. Mills, and S. R. P. Silva, "Charge Funneling through Metal Electrode Structuring for High-Efficiency Gains in Polymer Solar Cells," Adv. Electron. Mater., vol. 2, no. 9, p.
1600049, Sep. 2016.

[24] G. A. J. Amaratunga and S. R. P. Silva, "Nitrogen containing hydrogenated amorphous carbon for thin-film field emission cathodes," Appl. Phys. Lett., vol. 68, no. 18, p. 2529, Apr. 1996.

[25] J. D. Carey, R. D. Forrest, and S. R. P. Silva, "Origin of electric field enhancement in field emission from amorphous carbon thin films," Appl. Phys. Lett., vol. 78, no. 16, p. 2339, Apr. 2001.

[26] A. H. Fallahpour, S. Kienitz, and P. Lugli, "Origin of Dark Current and Detailed Description of Organic Photodiode Operation Under Different Illumination Intensities," IEEE Trans. Electron Devices, vol. 64, no. 6, pp. 2649-2654, Jun. 2017.

[27] J. W. Kingsley, S. J. Weston, and D. G. Lidzey, "Stability of XRay Detectors Based on Organic Photovoltaic Devices," IEEE J. Sel. Top. Quantum Electron., vol. 16, no. 6, pp. 1770-1775, Nov. 2010.

[28] D. Marre and G. Marinello, "Comparison of $p$-type commercial electron diodes for in vivo dosimetry," Med. Phys., vol. 31, no. 1, pp. 50-56, Dec. 2003.

[29] H. Song, M. Ahmad, J. Deng, Z. Chen, N. J. Yue, and R. Nath, "Limitations of silicon diodes for clinical electron dosimetry," Radiat. Prot. Dosimetry, vol. 120, no. 1-4, pp. 56-59, Sep. 2006.

[30] Y. Shimohigashi et al., "Angular dependence correction of MatriXX and its application to composite dose verification," $J$. Appl. Clin. Med. Phys., vol. 13, no. 5, pp. 198-214, Sep. 2012.

[31] S. M. Jafari et al., "Low-cost commercial glass beads as dosimeters in radiotherapy," Radiat. Phys. Chem., vol. 97, pp. 95-101, Apr. 2014.

[32] I. Griessbach, M. Lapp, J. Bohsung, G. Gademann, and D. Harder, "Dosimetric characteristics of a new unshielded silicon diode and its application in clinical photon and electron beams," Med. Phys., vol. 32, no. 12, pp. 3750-3754, Nov. 2005.

[33] J. R. Kerns, S. F. Kry, N. Sahoo, D. S. Followill, and G. S. Ibbott, "Angular dependence of the nanoDot OSL dosimeter," Med. Phys., vol. 38, no. 7, pp. 3955-3962, Jun. 2011.

[34] N. Kadoya et al., "Dosimetric properties of radiophotoluminescent glass detector in low-energy photon beams," Med. Phys., vol. 39, no. 10, pp. 5910-5916, Sep. 2012.

[35] P. A. Jursinic, "Characterization of optically stimulated luminescent dosimeters, OSLDs, for clinical dosimetric measurements," Med. Phys., vol. 34, no. 12, pp. 4594-4604, Nov. 2007.

[36] E. Roessl, J.-P. Schlomka, and R. Proksa, "Edge-on semiconductor X-ray detectors - towards high-rate counting computed tomography," in 2008 IEEE Nuclear Science Symposium Conference Record, 2008, pp. 1748-1751.

[37] S. Yoshida and T. Ohsugi, "Application of silicon strip detectors to X-ray computed tomography," Nucl. Instruments Methods Phys. Res. Sect. A Accel. Spectrometers, Detect. Assoc. Equip., vol. 541, no. 1-2, pp. 412-420, Apr. 2005.

[38] F. Arfelli et al., "Design and evaluation of AC-coupled, FOXFET-biased, 'edge-on' silicon strip detectors for X-ray imaging," Nucl. Instruments Methods Phys. Res. Sect. A Accel. Spectrometers, Detect. Assoc. Equip., vol. 385, no. 2, pp. 311320, Jan. 1997. 